\title{
Zoonotic transmission of Teladorsagia circumcincta and Trichostrongylus species in Guilan province, northern Iran: molecular and morphological characterizations
}

\author{
Keyhan Ashrafi ${ }^{1}$, Meysam Sharifdini ${ }^{1 *}$ (D), Zahra Heidari ${ }^{2}$, Behnaz Rahmati ${ }^{1}$ and Eshrat Beigom Kia ${ }^{3}$
}

\begin{abstract}
Background: Parasitic trichostrongyloid nematodes have a worldwide distribution in ruminants and frequently have been reported from humans in Middle and Far East, particularly in rural communities with poor personal hygiene and close cohabitation with herbivorous animals. Different species of the genus Trichostrongy/us are the most common trichostrongyloids in humans in endemic areas. Also, Ostertagia species are gastrointestinal nematodes that mainly infect cattle, sheep and goats and in rare occasion humans. The aim of the present study was to identify the trichostrongyloid nematodes obtained from a familial infection in Guilan province, northern Iran, using morphological and molecular criteria.

Methods: After anthelmintic treatment, all fecal materials of the patients were collected up to $48 \mathrm{~h}$ and male adult worms were isolated. Morphological identification of the adult worms was performed using valid nematode keys. Genomic DNA was extracted from one male worm of each species. PCR amplification of ITS2-rDNA region was carried out, and products were sequenced. Phylogenetic analysis of the nucleotide sequence data was performed using MEGA 6.0 software.
\end{abstract}

Results: Adult worms expelled from the patients were identified as T. colubriformis, T. vitrinus and Teladorsagia circumcincta based on morphological characteristics of the males. Phylogenetic analysis illustrated that each species obtained in current study was placed together with reference sequences submitted to GenBank database.

Conclusions: The finding of current study confirms the zoonotic aspect of Trichostrongylus species and $T$. circumcincta in inhabitants of Guilan province. The occurrence of natural human infection by T. circumcincta is reported for the first time in Iran and the second time in the world.

Keywords: Trichostrongylus colubriformis, Trichostrongylus vitrinus, Teladorsagia circumcincta, Human, Iran

\section{Background}

Trichostrongyloid nematodes (Nematoda: Trichostrongyloidae) are significant parasites of the digestive tract of herbivorous animals. Various genera of these nematodes are prevalent in ruminants in different countries throughout the world [1]. They have an important veterinary role due to their impact on animal health and severe economic losses in herbivores [1]. Among the

\footnotetext{
* Correspondence: sharifdini@gums.ac.ir; sharifdini5@gmail.com

${ }^{1}$ Department of Medical Parasitology and Mycology, School of Medicine,

Guilan University of Medical Sciences, Rasht, Iran

Full list of author information is available at the end of the article
}

Trichostrongyloidae, Trichostrongylus is the most common genus infecting humans in endemic regions [1]. Other genera of trichostrongyloid nematodes such as Haemonchus contortus, Marshallagia marshalli, Nematodirus abnormalis, Ostertagia ostertagi and O. circumcincta (Teladorsagia circumcincta) have been reported in humans, particularly in Iran and Azerbaidjan, of former Soviet Union [2-5].

Trichostrongylus species are primarily parasites of livestock with a worldwide distribution but they have also been reported from humans in Middle and Far East and Africa, particularly in rural communities with poor

(c) The Author(s). 2020 Open Access This article is distributed under the terms of the Creative Commons Attribution 4.0 International License (http://creativecommons.org/licenses/by/4.0/), which permits unrestricted use, distribution, and reproduction in any medium, provided you give appropriate credit to the original author(s) and the source, provide a link to the Creative Commons license, and indicate if changes were made. The Creative Commons Public Domain Dedication waiver (http://creativecommons.org/publicdomain/zero/1.0/) applies to the data made available in this article, unless otherwise stated. 
personal hygiene and close cohabitation with herbivorous animals [2, 4, 6-8]. Epidemiological studies have demonstrated trichostrongylosis as a prevalent parasitic infection in domestic ruminants and humans in different regions of Iran [2, 9]. Prevalences of 53.6, 46.8, 42.4, 38.7, 37 and $35.8 \%$ have been reported in ruminants from Isfahan, Khuzestan, Mazandaran, Kermanshah, Hormozgan and West Azerbaijan provinces and 71, 67, 42, 37, 19, 8, 2 and 1\% in humans in Khuzestan, Isfahan, Tehran, Hormozgan, Kermanshah, Mazandaran, West Azerbaijan and Sistan \& Baluchestan provinces in the past decades respectively [2]. Recent studies indicate a decreasing trend in the prevalences of trichostrongylosis in both animals and humans in Iran. However, human trichostrongylosis is still a health problem in some regions of the country. The prevalences of $18.1 \%$ [9], $3.05 \%$ [6] and 2.1\% [10] have been reported from Khouzestan, Guilan, and Mazandaran provinces in recent years respectively.

Until now, ten species of the genus Trichostrongylus have been reported from humans in various parts of Iran, which is unique in the world when the number of collected species is taken into consideration. Among these species, T. colubriformis and T. orientalis were more common in past decades in the country $[2,6,11-13]$, but recent studies in the north of Iran have shown $T$. colubriformis as the predominant species in infected individuals $[6,7,14,15]$. Humans mostly acquire the infection through consumption of fresh vegetables contaminated with infective filariform larvae [6, 7]. Trichostrongylosis is usually asymptomatic in low intensity infections, but abdominal pain, diarrhea, anorexia, nausea, weakness, mild anemia, low-grade peripheral eosinophilia, pulmonary and cutaneous symptoms are the most common manifestations in heavy infections $[8,16,17]$.

Ostertagia species, commonly known as the brown stomach worm, are gastrointestinal nematodes that infect cattle, sheep and goats and other wild ruminants worldwide, especially in temperate and cool climates. O. ostertagi mainly infects cattle, but also sheep, goats and other domestic and wild ruminants [1]. It has been reported from two persons in central part of Iran and one person in Azerbaijan of former Soviet Union [3]. T. circumcincta, which was originally placed in the genus Ostertagia, is a parasitic nematode of gastric glands of the stomach that infects sheep and goats all around the world and leads to weight loss, decreased wool production and death [18]. The prevalence rates of 17.3 to $47.2 \%$ for T. circumcincta have been reported in sheep and goats in different provinces of Iran [19-21]. A single human case of T. circumcincta has been reported from Azerbaijan of former Soviet Union in the past decades [5].

Detection of characteristic eggs in stool samples of humans and animals is a routine diagnostic method for trichostrongyloid infections [22]. However, classification of Trichostrongylus species and other genera of trichostrongyloid nematodes are based on differences in the morphology of male worms [19].

Recently, PCR-based tools are applied for reliable identification and phylogenetic analysis of nematodes. Based on the recent studies, the internal transcribed spacer 2 (ITS2) region of ribosomal DNA is a useful tool for analyzing genetic variations and phylogenetic relationships in nematodes belonging to the Trichostrongylidae family $[6,7,20,21]$. The aim of the present study was identification, molecular characterization and phylogenetic analysis of human specimens of Trichostrongylus and Teladorsagia obtained from the patients during a familial infection in Langroud district of Guilan province based on ITS2 region of ribosomal DNA.

\section{Methods}

\section{Study area}

Guilan province is one of the 31 provinces of Iran located at the southern littoral of Caspian Sea in northern Iran and is comprised of sixteen districts. Langroud district with an area of $480 \mathrm{~km}^{2}\left(37^{\circ} 11^{\prime} 49^{\prime \prime} \mathrm{N}, 50^{\circ} 9^{\prime} 13^{\prime \prime} \mathrm{E}\right)$ is located at the eastern part of the province (Fig. 1). Overall, 140,686 (70,675 males and 70,011females) were recorded to live in 49,351 families in the study region in 2016.

\section{Sample collection and morphological identification}

Seven members of a family living in Langroud district, located at the eastern part of Guilan province, were diagnosed to have trichostrongylosis following comprehensive clinical and laboratory examinations. Abdominal, epigastric and right upper quadrant pain, diarrhea, low grade fever, rigors especially at nights, allergic manifestations including urticaria associated with itching, flatulence and weight loss were the most important clinical manifestations declared by the patients. Hypereosinophilia ranging from 25 to $60 \%$ were seen. No parasite ova were obtained following examination of three stool samples on every other day using formalin-ether and KatoKatz techniques. Since the patients were living in a highrisk area of human fasciolosis they were treated for acute disease using Egaten $(10 \mathrm{mg} / \mathrm{kg})$. No evidence of treatment success was observed about four weeks after drug therapy. Surprisingly, all patients shed Trichostrongylus ova following a new set of three stool examination. The infected individuals were treated with a single dose of albendazole $(400 \mathrm{mg})$ followed by $200 \mathrm{mg}$ of mebendazole per day for 3 days [14]. All fecal materials of the patients was collected up to $48 \mathrm{~h}$ following chemotherapy in large screw capped plastic containers in $70 \%$ alcohol. Fecal materials were homogenated slowly and filtered using tap water over a sieve of 100 mesh $(150 \mu \mathrm{m})$ for collecting the adult worms. The 


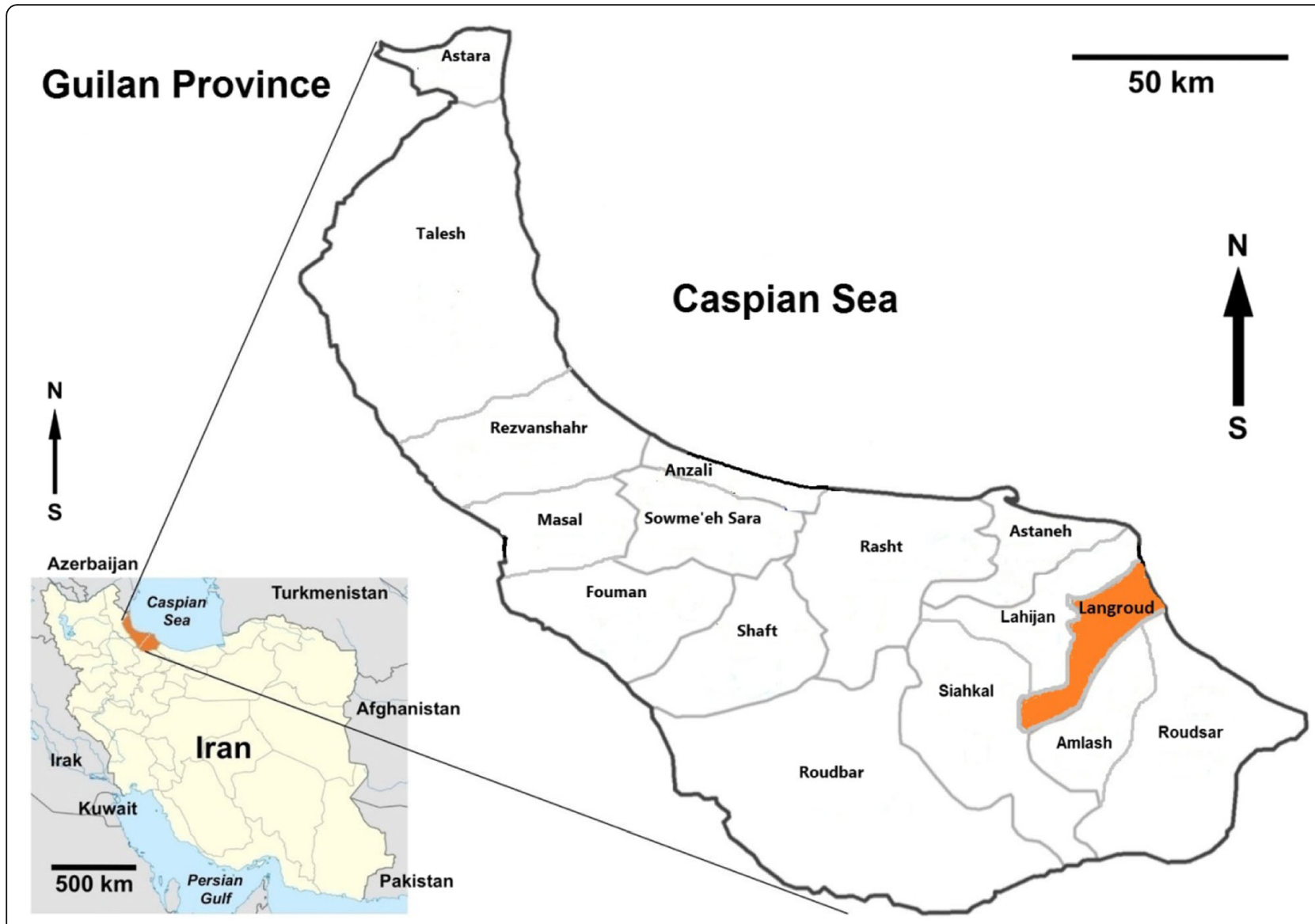

Fig. 1 Map of Iran showing geographical location of Guilan province and the study area, Langroud district (https://ars.els-cdn.com/content/image/1-s2.0-S2352340918313313 gr1.jpg)

isolated worms were put in 70\% alcohol for morphological and molecular studies. Morphological identification of the adult worms was performed by putting the males in a mixture of lactophenol and azocarmine on a glass slide, using valid nematodes systematic keys [23]. The specimens were also photographed using a light microscope equipped with a digital camera (TUCSEN, TrueChrome II, China).

\section{DNA isolation and PCR amplification}

A single male worm of each species isolated from the patients was processed for DNA extraction. The worms were washed with distilled water to remove ethanol and genomic DNA was extracted using Bioneer DNA extraction kit (Bioneer Corporation, Daejeon, Korea) according to the manufacturer's instructions and stored at $-20^{\circ} \mathrm{C}$ until PCR amplification.

PCR reactions were performed in $30 \mu \mathrm{L}$ volumes containing $2 \times$ red PCR premix (Ampliqon, Odense, Denmark), 20 pmol of each primer and $3 \mu \mathrm{L}$ of extracted DNA. Primers NC1 (5-ACGTCTGGTTCAGGGTTGTT-3) and NC2 (5TTAGTTTCTTTTCCTCCGCT-3) amplify a 328 base pair (bp) target of internal transcribed spacer 2 (ITS2) gene [24]. The thermal PCR profiles included an initial denaturation step at $95^{\circ} \mathrm{C}$ for $6 \mathrm{~min}$, followed by 35 cycles of denaturation at $94^{\circ} \mathrm{C}$ for $45 \mathrm{~s}$, annealing at $60^{\circ} \mathrm{C}$ for $90 \mathrm{~s}$, extension at $72{ }^{\circ} \mathrm{C}$ for $60 \mathrm{~s}$, followed by a final extension at $72{ }^{\circ} \mathrm{C}$ for 5 min. Samples containing water instead of templates were used as negative control. DNA extracted from T. colubriformis isolate of sheep was used as positive control in PCR reaction. The PCR products were electrophoresed on a $1.5 \%$ of agarose gel and visualized using ethidium bromide on an UV Transilluminator and digitally photographed.

\section{Sequencing and phylogenetic analysis}

PCR products were purified using a commercial purification kit (Bioneer, South Korea), according to the manufacturer's instructions. Purified products were sequenced in both directions using identical forward and reverse primers as in the PCR. Sequence results were edited and analyzed by BioEdit software (http://www.mbio.ncsu.edu/bioedit/ bioedit.html), and consensus sequences were compared with GenBank reference sequences using the BLAST system (http://www.ncbi.nlm.nih.gov/). The sequences of $T$. 
colubriformis, T. vitrinus and T. circumcincta were deposited in GenBank database under the accession numbers KF826913, KF872228 and KF989498, respectively.

Phylogenetic analysis was carried out using sequences obtained in this study along with relevant sequences deposited in the GenBank. A maximum likelihood tree was constructed based on the Tamura-Nei model and pairwise comparisons were made of the level of sequence differences within and among species using the MEGA 6.0 software. Bootstrap analysis was performed to determine the robustness of the finding based on 1000 replications.

\section{Results}

\section{Parasitological findings}

Adult worms isolated from fecal materials of the patients were identified as $T$. colubriformis, $T$. vitrinus and $T$. circumcincta according to the morphology of bursa copulatrix and spicules of the males. The morphological characteristics of spicules and gubernaculum of T. colubriformis, $T$. vitrinus and $T$. circumcincta are illustrated in Fig. 2. Based on systematic references [23], a brief description of spicules of male worms which have been collected from the patients is presented here.

- T. colubriformis: Spicules are slightly unequal in lengths and have boat-shaped structure with a thick outgrowth capping at proximal end. Also the gubernaculum has a wavy curve with two bends when seen laterally.

- T. vitrinus: The spicules are small, near equal and straight with sharply tapering at distal ends.
- T. circumcincta: The spicules are equal and each one fitted two fins at the sides. The posterior end of the spicule is split into two branches of equal length.

Table 1 shows the information of the patients such as number and species of the male worms collected by parasitological method in each treated patient.

\section{Molecular findings}

A $328 \mathrm{bp}$ segment of the ITS2 gene was successfully amplified from each of the male worms (Fig. 3). The sequences were compared with different sequences available in GenBank database, by using the BLAST system.

The molecular and phylogenetic analysis confirmed the morphologically distinguished worms. Phylogenetic tree indicates that each species obtained in current study is placed together with the same submitted to GenBank database (Fig. 4).

The MEGA 6 software was used to calculate mean intraspecies distance. The mean intra-species distance rate within specimens of $T$. colubriformis, T. vitrinus and T. circumcincta obtained in current study and those available in GenBank amounted to $0.7,1.1,1.5 \%$, respectively; meanwhile, mean inter-species sequence differences among subfamily Trichostrongylinae and also between subfamily Ostertaginae were significantly higher, being 2.6 and $6.5 \%$, respectively.

\section{Discussion}

Human infection with Trichostrongylus species is common in many countries throughout the world, in particular those located in the Middle East, Far East, and
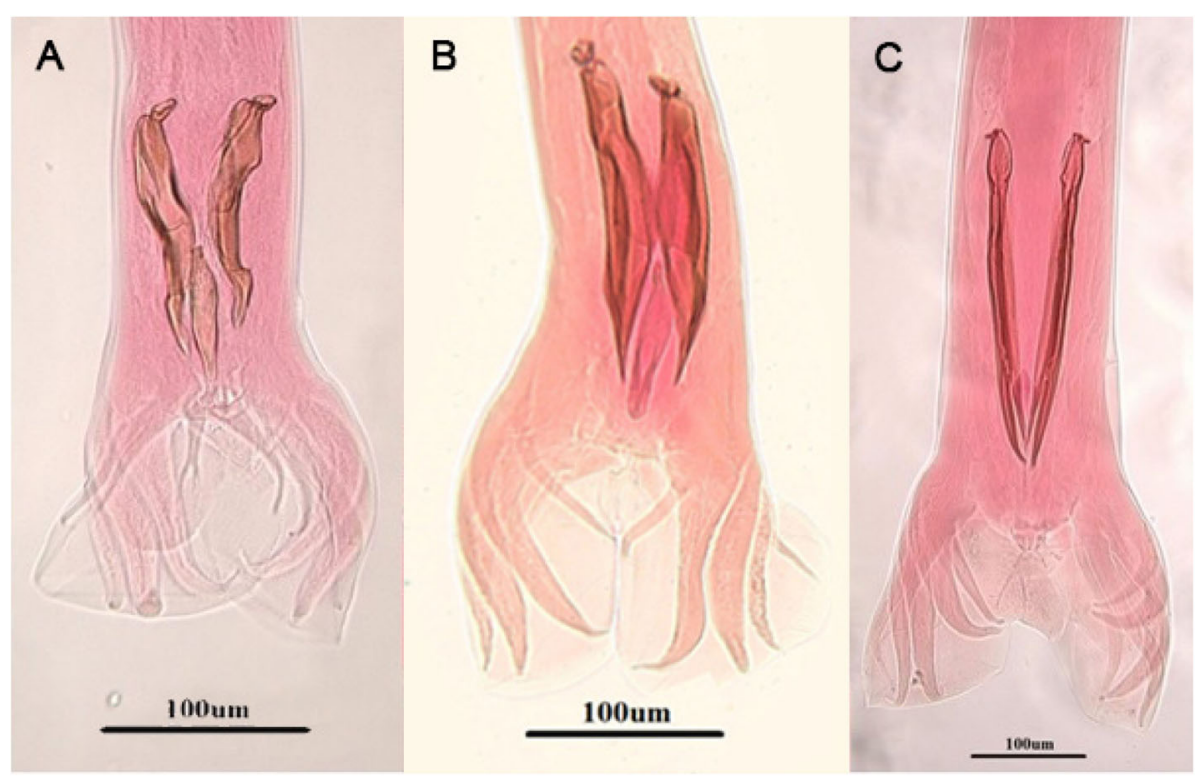

Fig. 2 Light microscope view of trichostrongylid nematodes isolated from humans in Langroud district of Guilan province, northern Iran. Copulatory bursa and spicules of Trichostrongylus colubriformis $\mathbf{a}$, T. vitrinus $\mathbf{b}$ and Teladorsagia circumcincta c (Scale bar: $100 \mu \mathrm{m}$ ) 
Table 1 Number and species of the male worms collected from seven infected individuals in Langroud district, Guilan province, northern Iran. Ova* ${ }^{*}$ presence of ova before treatment; Ova** $=$ presence of ova after treatment

\begin{tabular}{|c|c|c|c|c|c|c|}
\hline \multirow[t]{2}{*}{ Patients } & \multirow[t]{2}{*}{ ova* } & \multirow[t]{2}{*}{ ova** } & \multicolumn{4}{|c|}{ Number of male worms collected following treatment } \\
\hline & & & Trichostrongylus colubriformis & Trichostrongylus vitrinus & Teladorsagia circumcincta & Tota \\
\hline 1 & + & - & 14 & 3 & - & 17 \\
\hline 2 & + & - & 19 & 5 & 2 & 26 \\
\hline 3 & + & - & - & - & - & - \\
\hline 4 & + & - & 8 & - & - & 8 \\
\hline 5 & + & - & 11 & 5 & 2 & 18 \\
\hline 6 & + & - & - & - & - & - \\
\hline 7 & + & - & 7 & 4 & - & 11 \\
\hline
\end{tabular}

some African countries [5]. It has also a long history in Iran with the highest infection rates in the world that is most probably due to people's habit of living in close contact with their livestock in rural regions and nomadic style of life in some parts of the country [2]. In addition, use of animal feces as fertilizer, consuming fresh wild grown aromatic plants, collecting from environment by villagers, as a part of human diet in many endemic areas and preparing parts of needed fuel from animal dung could be regarded among the other possible risk factors of human trichostrongylosis in endemic areas of Iran.

In recent years, the prevalence of most human geohelminths especially Ascaris lumbricoides and hookworms are sharply decreased over time in Iran [25, 26]; however, Strongyloides stercoralis [27-29] and Trichostrongylus spp. [6, 7, 14] are still reporting in some parts of the country. In 1961 prevalences of ascariasis in Kermanshah, Kurdistan, Isfahan, Guilan, Mazandaran, Khuzestan, West Azerbaijan and East Azerbayjan provinces were 69, 80, 80.6, 67.9, 86.3, 48.8 and $48.2 \%$ respectively. In all above mentioned areas the prevalence of ascariasis has decreased to less than $1 \%$ at present time. For example, In Mazandaran province, the prevalence of the disease in 1961, 1974, 1992, 2004, 2012 and 2016 have been reported as $86.3,23,1.2,0.3,0.4$ and $0.6 \%$ respectively $[25,30,31]$.

The same situation is seen in other parts of the country and a decreasing trend is also present for other geohelminthes. On the other hand, very rare cases of hookworm infection and ascariasis are reported in recent years while cases of strongyloidosis and trichostrongylosis are more encountered in some regions especially in northern provinces. The prevalences of $9.7 \%$ [32], 4.9\% [33] and 0.9\% [34] have been reported for strongyloidosis in Khuzestan, Mazandaran and Guilan provinces, respectively. In a different study carried out in Guilan province, $42 \%$ of hypereosinophilic individuals referred to the parasitology laboratory of Faculty of Medicin of Guilan University of Medical Sciences were found to be infected with S. stercoralis [35]. In addition, various studies verify the presence of human cases of trichstrongylosis in Iran in recent years. According to these studies the prevalences of human trichstrongylosis vary from 0.4 to $18 \%$ in some parts of the country $[6,9,10,30]$.

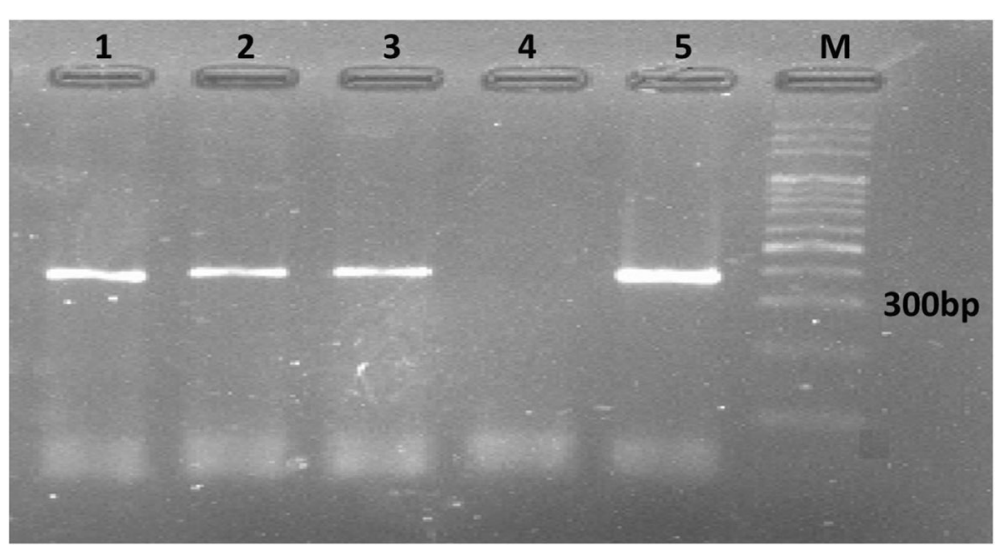

Fig. 3 Agarose-gel electrophoresis of polymerase chain reaction (PCR) products amplified with genomic DNA from trichostrongylid nematodes worms obtained in current study. Lane 1: PCR products of Trichostrongylus colubriformis; Lane 2: T. vitrinus; Lane 3: Teladorsagia circumcincta; Lane 4: Negative control; Lane 5: Positive control; and Lane M: 100-bp DNA marker 




Fig. 4 Phylogenetic tree of isolates of Trichostrongylus spp. and Teladorsagia circumcincta obtained in current study ( $\mathbf{\Delta}$ ) and reference sequences from previous studies based on ITS2 nucleotide sequences and constructed using Tamura-Nei model in MEGA software version 6. Iran (IRN), Laos (LAO), Russia (RUS), United States (USA), United Kingdom (GBR), Australia (AUS), India (IND), Uzbekistan (UZB) and New Zealand (NZL) are represented with country codes (ISO 3166-1 a-3 codes)

Infection with ten species of Trichostrongylus including T. colubriformis [2], T. orientalis [2], T. vitrinus [2], T. skrjabini [2], T. axei [2], T. probolurus [2], T. lerouxi [12], T. capricola [13], T. longispicularis [6], and an unnamed species [36] have been reported from humans in different parts of Iran. In the past decades, predominant species infecting humans were T. orientalis and T. colubriformis in most areas of the country such as central and southwestern parts. T. orientalis had low prevalence and intensity in herbivorous animals in these regions, which suggests that this species is predominantly a human parasite which is also prevalent in Far East, in particular in Japan, Korea and China [2, 37].

The using of human excreta, or night soil as fertilizer in agriculture in the past might be responsible for high prevalence of $T$. orientalis in these regions because the transmission of this parasite is mainly from human to human [2]. On the other hand, T. colubriformis was second most common species among inhabitants in the areas of Iran where it was also the most prevalent in domestic animals, and consequently can be considered to be mainly a zoonotic species [2]. Currently, it is not 
clearly known which species is prevalent among individuals in these regions.

The prevalence of T. vitninus in animals has been higher than that of $T$. colubriformis in most parts of the country, while the rates of trichostrongylosis due to $T$. colubniformis in humans have been reported to be higher than those due to T. vitninus [2]. It might be ascribed to higher susceptibility of humans to T. colubriformis. In north of Iran, T. colubriformis followed by $T$. axei and T. vitrinus, were reported most frequently in infected individuals in the past whereas the number of T. orientalis was usually low [2]. In these patients, the predominant species with high intensity was $T$. colubriformis. Gholami et al. (2015) and Sharifdini et al. (2017) have identified $T$. colubriformis as the most probable common species and T. axei in human in Mazandaran province, north of Iran using DNA detection in stool samples [15]. Recently, Sharifdini et al. (2017) reported T. colubriformis, as predominant species, T. vitrinus, $T$. axei and T. longispicularis in inhabitants of an endemic area in Guilan province. In the recent study, the authors reported the occurrence of natural human infection by T. longispicularis for the first time in the world [6]. Also, T. colubriformis was predominant among Trichostrongylus species infecting humans in Thailand [38], France [39] and Laos [4, 8]. This may be due to high prevalence of this species in herbivorous animals and also its high zoonotic potential [2]. Similar to the present study, human infections with $T$. vitrinus had been reported in the past from most parts of Iran, Armenia and Egypt [2]. Recently, T. vitrinus were detected in inhabitants of Fouman district in Guilan province [6].

Members of Ostertagia genus are found in the abomasum of cattle, sheep, goats and cervids worldwide. $O$. ostertagi is a cosmopolitan parasite of cattle. It is found with a lesser extent in sheep, goats, wild ruminants and horses $[1,40]$. It was also reported occasionally in humans from Iran and Azerbaidjan [3]. T. circumcincta is the most important species of the genus found in sheep but it occurs in a variety of other ruminants such as goats and camels. This species has been reported from sheep, goats, wild sheep and camels in different parts of Iran [1, 18, 4144]. Naem et al. [41] showed that 38\% of sheep of Mazandaran province, located at the eastern part of Guilan, were infected with this parasite [41]. T. circumcincta has been reported only once to infect an inhabitant in Azerbaijan of former Soviet Union and hence this is the second report of human infection with this parasite [5].

Molecular phylogenetic analysis is a helpful tool to gain information on evolutionary relationships between the organisms. The existence of genetic variation among Trichostrongylus nematodes has been confirmed previously $[6,7,20]$. There are a few studies which have analyzed molecular-phylogenetic characterization of human trichostrongylosis $[6,7,45,46]$. In this study, phylogenetic analysis represented that all Trichostrongylus species and $T$. circumcincta were clearly separated and there were only few differences among the species isolated from our study and those obtained from human and livestock subjects in other countries. Sequence of $T$. colubriformis specimen obtained in current study is placed in one branch together with isolates from Iran obtained from human (KP663664 and KY355022) and sheep (KJ755060), Laos (AB503247) goat isolate, Laos (AB503250) human isolate, Australia (KC521370) rabbit isolate and USA isolate from cattle (KP150536).

Furthermore, the phylogenetic analysis shows that $T$. vitrinus specimen of the current study has the same sequence with $T$. vitrinus obtained from human (KY355027) and sheep (KF880745) in Iran, and also sheep from Great Britain (AY439027) and New Zealand (KC998731). On the other hand, the phylogenetic tree represents that $T$. circumcincta specimens obtained in current study is placed together with sheep isolate from Iran (JQ989274). Although, the phylogenetic tree indicates that $T$. colubriformis, T. vitrinus and T. circumcincta are placed in separate branches, nevertheless, there is a high homology between sequences of human and livestock isolates. This result along with the other epidemiological and molecular studies reveals that zoonotic transmission is the most common route for these species.

\section{Conclusions}

The finding of current study verifies the presence of Trichostrongylus species and T. circumcincta in inhabitants of Guilan province. Phylogenetic analysis shows that Trichostrongylus species and T. circumcincta isolated from human and animal have close associations and this verifies livestock as the main source of human infections. Consumption of fresh contaminated vegetables, close contact with livestock and their fecal materials and lack of personal hygiene in some rural regions might be the most recognized risk factors in the study area. Considering the current trend in growing use of animal dung as fertilizer in organic farms, the incidence of human infections with trichostrongyloid nematodes may increase in the future. In present study, the occurrence of natural human infection by $T$. circumcincta is reported for the first time in Iran and the second time in the world.

\section{Abbreviations \\ ITS2: Internal transcribed spacer 2; PCR: Polymerase Chain Reaction; rDNA: Ribosomal DNA}

\section{Acknowledgments}

The authors would like to thank all people who have contributed to this research. 


\section{Authors' contributions}

$\mathrm{KA}, \mathrm{EBK}$ and MSH designed the study. KA, ZH, BR and MSH carried out the experiments. KA and MSH assisted with manuscript writing. All authors read and approved the final version of the manuscript.

\section{Funding}

Not applicable.

\section{Availability of data and materials}

The data supporting the conclusion of this article are included within the text and additional files. The sequences were deposited in GenBank database under the accession numbers KF826913, KF872228 and KF989498.

\section{Ethics approval and consent to participate}

The study was approved by Ethics Committee of Guilan University of Medical Sciences, Iran (Ref. No. IR.GUMS.REC.1398.423). Written informed consent was attained for each patient and confidentiality of the information was assured. The results of the diagnostic methods of the patients were delivered to them in confidential written format and all their diagnostics tests were free of charge.

\section{Consent for publication}

Not applicable.

\section{Competing interests}

The authors declare that they have no competing interests.

\section{Author details}

'Department of Medical Parasitology and Mycology, School of Medicine, Guilan University of Medical Sciences, Rasht, Iran. ${ }^{2}$ Department of Medical Microbiology and Parasitology, School of Medicine, Ardabil University of Medical Sciences, Ardabil, Iran. ${ }^{3}$ Department of Medical Parasitology and Mycology, School of Public Health, Tehran University of Medical Sciences, Tehran, Iran.

Received: 23 December 2018 Accepted: 31 December 2019

Published online: 10 January 2020

\section{References}

1. Roberts $L S$, Janovy J. Foundations of parasitology. 8th ed. New York: McGraw-Hill; 2012

2. Ghadirian E, Arfaa F. Present status of trichostrongyliasis in Iran. Am J Trop Med Hyg. 1975;24:935-41.

3. Ghadirian E, Arfaa F. First report of human infection with Haemonchus contortus, Ostertagia ostertagi, and Marshallagia marshalli (family Trichostrongylidae) in Iran. J Parasitol. 1973;59:1144-5.

4. Sato M, Yoonuan T, Sanguankiat S, et al. Short report: human Trichostrongylus colubriformis infection in a rural village in Laos. Am J Trop Med Hyg. 2011;84:52-4.

5. Muller R. Worms and human disease. 2nd ed. London: CABl; 2002. 138 p.

6. Sharifdini M, Derakhshani S, Alizadeh SA, et al. Molecular identification and phylogenetic analysis of human Trichostrongylus species from an endemic area of Iran. Acta Trop. 2017;176:293-9.

7. Sharifdini M, Heidari Z, Hesari Z, et al. Molecular phylogenetics of Trichostrongylus species (Nematoda: Trichostrongylidae) from humans of Mazandaran province, Iran. Korean J Parasitol. 2017;55:279-85.

8. Watthanakulpanich D, Pongvongsa T, Sanguankiat S, et al. Prevalence and clinical aspects of human Trichostrongylus colubriformis infection in Lao PDR. Acta Trop. 2013:126:37-42.

9. Farahnak A. Survey on prevalence of parasites in the central area of Khouzestan. Med J Tabriz Univ Med Sci Health Serv. 2001;35:57-62.

10. Daryani A, Sharif M, Nasrolahei M, et al. Epidemiological survey of the prevalence of intestinal parasites among schoolchildren in Sari, northern Iran. Trans R Soc Trop Med Hyg. 2012;106:455-9.

11. Ghasemikhah R, Mirhendi H, Kia E, et al. Morphological and morphometrical description of Trichostrongylus species isolated from domestic ruminants in Khuzestan province, Southwest Iran. Iran J Parasitol. 2011;6:82-8.

12. Ghadirian E. Human infection with Trichostrongylus lerouxi (Biocca, Chabaud, and Ghadirian, 1974) in Iran. Am J Trop Med Hyg. 1977;26:1212-3.

13. Ghadirian E, Arfaa F, Sadighian A. Human infection with Trichostrongylus capricola in Iran. Am J Trop Med Hyg. 1974;23:1002-3.
14. Ashrafi K, Tahbaz A, Sharifdini M, et al. Familial Trichostrongylus infection misdiagnosed as acute fascioliasis. Emerg Infect Dis. 2015;21:1869-70.

15. Gholami S, Babamahmoodi F, Abedian R, et al. Trichostrongylus colubriformis: possible most common cause of human infection in Mazandaran province, north of Iran. Iran J Parasitol. 2015;10:110-5.

16. Buonfrate D, Angheben A, Gobbi F, et al. Four clusters of Trichostrongylus infection diagnosed in a single center, in Italy. Infection. 2017;45:233-6.

17. Ghanbarzadeh L, Saraei M, Kia EB, et al. Clinical and haematological characteristics of human trichostrongyliasis. J Helminthol. 2018;93:149-53.

18. Craig TM. Gastrointestinal nematodes, diagnosis and control. Vet Clin North Am Food Anim Pract. 2018;34:185-99.

19. Lichtenfels JR, Hoberg EP, Zarlenga DS. Systematics of gastrointestinal nematodes of domestic ruminants: advances between 1992 and 1995 and proposals for future research. Vet Parasitol. 1997;72:225-38.

20. Ghasemikhah R, Sharbatkhori M, Mobedi I, et al. Sequence analysis of the second internal transcribed spacer (ITS2) region of rDNA for species identification of Trichostrongylus nematodes isolated from domestic livestock in Iran. Iran J Parasitol. 2012;7:40-6.

21. Sim KA, Hoar B, Kutz SJ, et al. Amplification of the second internal transcribed spacer ribosomal DNA of individual trichostrongylid nematode larvae by nested polymerase chain reaction. J Vet Diagn Investig. 2010:22:433-7.

22. Georgi J, McCulloch C. Diagnostic morphometry: identification of helminth eggs by discriminant analysis of morphometric data. P Helm Soc Wash. 1989;56:44-57.

23. Skrjabin, K.I., Shikhobalova, N.P., Shults, R.S. Essentials of nematodology. Trichostrongyloids of Animals and Man Vol. III Pub. House of Academy of Sciences, USSR, Moscow. 1954

24. Hoste H, Chilton NB, Gasser RB, et al. Differences in the second internal transcribed spacer (ribosomal DNA) between five species of Trichostrongylus (Nematoda: Trichostrongylidae). Int J Parasitol. 1995;25:75-80.

25. Rokni MB. The present status of human helminthic diseases in Iran. Ann Trop Med Parasitol. 2008;102:283-95.

26. Sharifdini M, Ghanbarzadeh L, Kouhestani-Maklavani N, et al. Prevalence and molecular aspects of human hookworms in Guilan province, northern Iran. Iran J Parasitol. 2017:12:374-81.

27. Sharifdini M, Kia EB, Ashrafi K, et al. An analysis of clinical characteristics of Strongyloides stercoralis in 70 indigenous patients in Iran. Iran J Parasitol. 2014:9:155-62.

28. Sharifdini M, Mirhendi $H$, Ashrafi $K$, et al. Comparison of nested polymerase chain reaction and real-time polymerase chain reaction with parasitological methods for detection of Strongyloides stercoralis in human fecal samples. Am J Trop Med Hyg. 2015;93:1285-91.

29. Sharifdini M, Hesari A, Mahdavi SA, et al. Strongyloides stercoralis hyperinfection in an unconscious diabetic patient with dermatomyositis. Indian J Pathol Microbiol. 2018;61:109-12.

30. Vahedi M, Gohardehi S, Sharif M, et al. Prevalence of parasites in patients with gastroenteritis at east of Mazandaran province, northern Iran. Trop Biomed. 2012;29:568-74.

31. Shahdoust S, Niyyati M, Haghighi A, et al. Prevalence of intestinal parasites in referred individuals to the medical centers of Tonekabon city, Mazandaran province. Gastroenterol Hepatol Bed Bench. 2016;9:75-9.

32. Sharifdini M, Keyhani A, Eshraghian MR, et al. Molecular diagnosis of strongyloidiasis in a population of an endemic area through nested-PCR. Gastroenterol Hepatol Bed Bench. 2018;11:68-74.

33. Kia EB, Mahmoudi M, Zahabiun F, et al. An evaluation on the efficacy of agar plate culture for detection of Strongyloides stercoralis. Iran J Parasitol. 2007;2:29-34

34. Asmar M, Ashrafi K, Amintahmasbi M, et al. Prevalence of intestinal parasitic infections in the urban areas of Bandar Anzali, northern Iran. J Guilan Univ Med Sci. 2014;22:18-25.

35. Ashrafi K, Tahbaz A, Rahmati B. Strongyloides stercoralis: the most prevalent parasitic cause of eosinophilia in Gilan province, northern Iran. Iran J Parasitol. 2010;5:40-7.

36. Ghadirian E, Biocca E. Trichostrongylus sp. from the intestine of man in Iran. Parassitologia. 1973;15:17-20.

37. John D, Petri W. Markell and Voge's medical parasitology. 9th ed. St. Louis, MO: Elsevier; 2006

38. Phosuk I, Intapan PM, Sanpool O, et al. Molecular evidence of Trichostrongylus colubriformis and Trichostrongylus axei infections in humans from Thailand and Lao PDR. Am J Trop Med Hyg. 2013;89:376-9.

39. Lattes $\mathrm{S}$, Ferte $\mathrm{H}$, Delaunay $\mathrm{P}$, et al. Trichostrongylus colubriformis nematode infections in humans, France. Emerg Infect Dis. 2011;17:1301-2. 
40. Williams JC, Knox JW. Epidemiology of Ostertagia ostertagi in warm temperate regions of the United States. Vet Parasitol. 1988;27:23-38,

41. Naem S, Gorgani T. Gastrointestinal parasitic infection of slaughtered sheep (Zel breed) in Fereidoonkenar city, Iran. Vet Res Forum. 2011;2:238-41.

42. Pestechian N, Kalani H, Faridnia R, et al. Zoonotic gastrointestinal nematodes (Trichostrongylidae) from sheep and goat in Isfahan, Iran. Acta Sci Vet. 2014;42:1-5.

43. Garedaghi Y, Hashemzadefarhang H, Fattahi A. Prevalence of abomasal nematodes in sheep slaughtered at Baneh town. Am J Anim Vet Sci. 2013;8:142-5.

44. Borji H, Razmi G, Movassaghi AR, et al. A study on gastrointestinal helminths of camels in Mashhad abattoir, Iran. Iran J Vet Res. 2010;11:174-9.

45. Phosuk I, Intapan PM, Prasongdee TK, et al. Human trichostrongyliasis: a hospital case series. Southeast Asian J Trop Med Public Health. 2015;46:191-7.

46. Yong TS, Lee JH, Sim S, et al. Differential diagnosis of Trichostrongylus and hookworm eggs via PCR using ITS-1 sequence. Korean J Parasitol. 2007;45: 69-74.

\section{Publisher's Note}

Springer Nature remains neutral with regard to jurisdictional claims in published maps and institutional affiliations.

Ready to submit your research? Choose BMC and benefit from:

- fast, convenient online submission

- thorough peer review by experienced researchers in your field

- rapid publication on acceptance

- support for research data, including large and complex data types

- gold Open Access which fosters wider collaboration and increased citations

- maximum visibility for your research: over $100 \mathrm{M}$ website views per year

At $\mathrm{BMC}$, research is always in progress.

Learn more biomedcentral.com/submissions 